\title{
STUDY OF THE RADIOACTIVITY INDUCED IN AIR BY A 15-MeV PROTON BEAM
}

\author{
S. Braccini ${ }^{1, *}$, A. Ereditato ${ }^{1}$, K. P. Nesteruk ${ }^{1}$, P. Scampoli ${ }^{1,2}$ and K. Zihlmann ${ }^{1}$ \\ ${ }^{1}$ Albert Einstein Center for Fundamental Physics, Laboratory for High Energy Physics, University of Bern, \\ Sidlerstrasse 5, Bern CH-3012, Switzerland \\ ${ }^{2}$ Dipartimento di Fisica, Università di Napoli Federico II, Complesso Universitario di Monte S. Angelo, \\ Napoli I-80126, Italy
}

*Corresponding author: saverio.braccini@lhep.unibe.ch

Received 29 January 2014; revised 28 May 2014; accepted 31 May 2014

\begin{abstract}
Radioactivity induced by a $15-\mathrm{MeV}$ proton beam extracted into air was studied at the beam transport line of the $18-\mathrm{MeV}$ cyclotron at the Bern University Hospital (Inselspital). The produced radioactivity was calculated and measured by means of proportional counters located at the main exhaust of the laboratory. These devices were designed for precise assessment of air contamination for radiation protection purposes. The main produced isotopes were ${ }^{11} \mathrm{C},{ }^{13} \mathrm{~N}$ and ${ }^{14} \mathrm{O}$. Both measurements and calculations correspond to two different irradiation conditions. In the former, protons were allowed to travel for their full range in air. In the latter, they were stopped at the distance of $1.5 \mathrm{~m}$ by a beam dump. Radioactivity was measured continuously in the exhausted air starting from 2 min after the end of irradiation. For this reason, the short-lived ${ }^{14} \mathrm{O}$ isotope gave a negligible contribution to the measured activity. Good agreement was found between the measurements and the calculations within the estimated uncertainties. Currents in the range of 120-370 nA were extracted in air for 10-30 s producing activities of 9-22 $\mathrm{MBq}$ of ${ }^{11} \mathrm{C}$ and ${ }^{13} \mathrm{~N}$. The total activities for ${ }^{11} \mathrm{C}$ and ${ }^{13} \mathrm{~N}$ per beam current and irradiation time for the former and the latter irradiation conditions were measured to be $(3.60 \pm 0.48) \times 10^{-3} \mathrm{MBq}(\mathrm{nA} \mathrm{s})^{-1}$ and $(2.89 \pm 0.37) \times 10^{-3} \mathrm{MBq}(\mathrm{nA} \mathrm{s})^{-1}$, respectively.
\end{abstract}

\section{INTRODUCTION}

The assessment of the radioactivity present in air is an important issue in radiation protection. A reliable and efficient monitoring is crucial for radioisotope production and research facilities. Typical examples are positron emission tomography (PET) radioisotope production centres, where ${ }^{41} \mathrm{Ar}$ is regularly produced in air during irradiation through the reaction ${ }^{40} \operatorname{Ar}(\mathrm{n}, \gamma)^{41} \mathrm{Ar}$. Furthermore, releases of $\beta^{+}$emitters in gas form (mainly ${ }^{13} \mathrm{~N}$ and ${ }^{18} \mathrm{~F}$ ) may provoke radiological hazards.

Some research applications require the extraction of the beam into air as, for example, PIXE and PIGE ion beam analysis and the bombardment of cell samples for radiation biology. For these kinds of experimental activities, radioisotopes are produced in air by nuclear reactions induced by the beam and an accurate assessment of the radioactivity is therefore needed.

In this work, a study of the radioactivity produced by a $15-\mathrm{MeV}$ proton beam extracted into air is presented. This work was performed at the cyclotron laboratory at the Bern University Hospital (Inselspital), which was realised for PET radioisotope production and multi-disciplinary research running in parallel ${ }^{(1,2)}$. This facility is equipped with a beam transport line ending in a separate bunker ${ }^{(3)}$, where the experiments reported in this study were performed. Specific detectors assure the continuous radiation protection monitoring of exhausted air. Low-sensitivity alarm detectors are located at the exhaust of each bunker and high-sensitivity proportional counters ${ }^{(4)}$ are placed at the main exhaust of the facility to assess the integral of the activity released into the atmosphere. These proportional counters were used to measure the radioactivity produced by extracting beams of known current into air. Calculations of the induced activity were performed and compared with the data.

\section{CALCULATION OF INDUCED RADIOACTIVITY}

When a $15-\mathrm{MeV}$ proton beam is extracted into air, radioactive isotope production occurs. The nuclei present in air can undergo nuclear reactions. Air is composed of nitrogen, oxygen, argon, carbon and traces of other elements. Nitrogen $(78.084 \%)$ and oxygen $(20.948 \%)$ represent by far the main components ${ }^{(5)}$. Concerning their isotope abundance, ${ }^{14} \mathrm{~N}$ accounts for $\sim 99.5 \%$ and ${ }^{16} \mathrm{O}$ for $\sim 99.8 \%$.

To assess the nuclear reactions, cross sections for the production of various isotopes by protons in air $^{(6)}$ together with target concentrations were considered. Two reactions were found to be dominant:

$$
\begin{aligned}
& { }^{14} \mathrm{~N}(p, \alpha){ }^{11} \mathrm{C}, \\
& { }^{16} \mathrm{O}(p, \alpha){ }^{13} \mathrm{~N} .
\end{aligned}
$$


Two further reactions involving ${ }^{14} \mathrm{~N}$ as a target were also considered due to their non-negligible cross section:

$$
\begin{aligned}
& { }^{14} \mathrm{~N}(p, d+p n){ }^{13} \mathrm{~N}, \\
& { }^{14} \mathrm{~N}(p, n){ }^{14} \mathrm{O} .
\end{aligned}
$$

Produced isotopes decay according to the following reactions:

$$
\begin{aligned}
& { }^{11} \mathrm{C} \rightarrow{ }^{11} \mathrm{~B}+e^{+}+v_{e}, \\
& { }^{13} \mathrm{~N} \rightarrow{ }^{13} \mathrm{C}+e^{+}+v_{e}, \\
& { }^{14} \mathrm{O} \rightarrow{ }^{14} \mathrm{~N}+e^{+}+v_{e} .
\end{aligned}
$$

Since the reactions are three-body decays, the maximum energies of the positron for ${ }^{11} \mathrm{C}$ and ${ }^{13} \mathrm{~N}$ are 0.96 and $1.19 \mathrm{MeV}$, respectively. For ${ }^{14} \mathrm{O}$, the maximum positron energy is $1.81 \mathrm{MeV}$ in $99.4 \%$ of the decays and $4.12 \mathrm{MeV}$ in the remaining $0.6 \%$.

The production rates of ${ }^{11} \mathrm{C},{ }^{13} \mathrm{~N}$ and ${ }^{14} \mathrm{O}$ are indicated as $P_{\mathrm{C}}, P_{\mathrm{N}}$ and $P_{\mathrm{O}}$, respectively. The production rate $P$ (in nuclei $\mathrm{s}^{-1}$ ) for one reaction is given by the formula:

$$
P=\frac{n \cdot I}{e} \int_{E_{\mathrm{i}}}^{E_{\mathrm{f}}} \frac{\sigma(E)}{\mathrm{d} E / \mathrm{d} x} \mathrm{~d} E,
$$

where $n$ is the target density (in nuclei $\mathrm{m}^{-3}$ ), $I$ the beam current, $e$ the elementary charge, $\sigma$ the reaction cross section, $E$ the energy of the incident particle and $x$ the travelled distance. The integral was calculated from the initial energy $E_{\mathrm{i}}$ of the proton to its final energy $E_{\mathrm{f}}$. Since protons are accelerated by the Bern cyclotron to the energy of $18 \mathrm{MeV}$ and passed through a $400-\mu \mathrm{m}$ thick aluminium exit window, the initial energy $E_{\mathrm{i}}$ of protons extracted into air was calculated by means of the simulation software $\operatorname{SRIM}^{(7)}$. $E_{\mathrm{i}}$ was found to be $15.56 \pm 0.10 \mathrm{MeV}$. In the case of the full proton range in air, $E_{\mathrm{f}}$ is equal to 0 . If the beam dump at $1.5 \mathrm{~m}$ from the exit window was used, $E_{\mathrm{f}}$ was also determined by SRIM. It was found to be $9.55 \pm 0.10 \mathrm{MeV}$. The reaction cross sections $\sigma(E)$ were evaluated on the basis of the measurements reported in refs ${ }^{(6,8,9)}$. The energy loss per unit length $\mathrm{d} E / \mathrm{d} x$ used for the evaluation of the integral was calculated by means of SRIM. Dry air near the sea level (International Commission on Radiation Units and Measurements-104) was considered. On this basis, the integral was computed numerically.

The resulting value $P$ was then used for the evaluation of the corresponding produced radioactivity. The number of radioactive nuclei $N$ present in air is a function of the irradiation time $t$ and is derived from the following equation:

$$
\frac{\mathrm{d} N(t)}{\mathrm{d} t}=-\lambda N(t)+P,
$$

where $\lambda$ is the decay constant of the particular isotope: $\lambda_{\mathrm{C}}=5.669 \times 10^{-4} \mathrm{~s}^{-1}, \lambda_{\mathrm{N}}=1.159 \times 10^{-3}$ $\mathrm{s}^{-1}$ and $\lambda_{\mathrm{O}}=9.818 \times 10^{-3} \mathrm{~s}^{-1}$ for ${ }^{11} \mathrm{C},{ }^{13} \mathrm{~N}$ and ${ }^{14} \mathrm{O}$, respectively. This leads to the expression for the activity $A$ at time $t$ :

$$
A(t)=P\left(1-\mathrm{e}^{-\lambda t}\right) .
$$

The results of the calculations for the full proton range in air as well as for protons stopped at the distance of $1.5 \mathrm{~m}$ are presented in Table 1 . For ${ }^{13} \mathrm{~N}$, the result is obtained by summing the rates given by the two production reactions. The production of ${ }^{11} \mathrm{C}$ was found to be dominant. The uncertainties of the performed calculations are due to the measured excitation functions ${ }^{(6,8,9)}$.

As shown in Figure 1, the contribution to the total activity due to ${ }^{14} \mathrm{O}$ rapidly decreases in time due to its

Table 1. Production rates per beam current are reported for full proton range and protons stopped at the distance of $1.5 \mathrm{~m}$.

\begin{aligned} & Full proton range \\ &$P_{\mathrm{C}} / I\left[\right.$ nuclei $\left.(\mathrm{nA} \mathrm{s})^{-1}\right](5.31 \pm 1.14) \times 10^{6} \\ & P_{\mathrm{N}} / I\left[\right.$ nuclei $\left.\left(\mathrm{nA} \mathrm{s}^{-1}\right)\right](8.15 \pm 1.28) \times 10^{5} \\ & P_{\mathrm{O}} / I\left[\right.$ nuclei $\left.(\mathrm{nA} \mathrm{s})^{-1}\right](2.40 \pm 1.16) \times 10^{5} \\ &$ Protons stopped at $1.5 \mathrm{~m} \\ & P_{\mathrm{C}} / I\left[\right.$ nuclei $\left.(\mathrm{nA} \mathrm{s})^{-1}\right](3.52 \pm 0.66) \times 10^{6} \\ & P_{\mathrm{N}} / I\left[\right.$ nuclei $\left.(\mathrm{nA} \mathrm{s})^{-1}\right](7.56 \pm 1.01) \times 10^{5} \\ & P_{\mathrm{O}} / I\left[\right.$ nuclei $\left.(\mathrm{nA} \mathrm{s})^{-1}\right](1.72 \pm 0.82) \times 10^{5} \\ &$\hline\end{aligned}

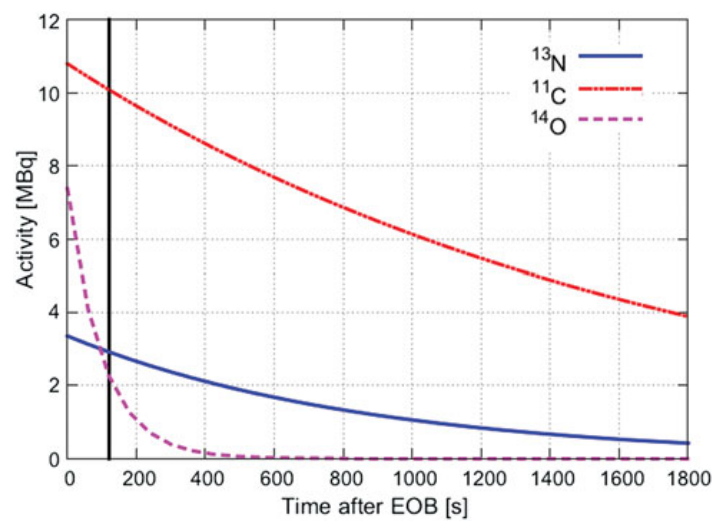

Figure 1. Time evolution of the total calculated activity for ${ }^{11} \mathrm{C},{ }^{13} \mathrm{~N}$ and ${ }^{14} \mathrm{O}$ in the full proton range irradiation condition. The EOB is represented by $t=0$. The vertical line corresponds to the start of the measurements, 2 min after EOB. A very similar plot was obtained for the other irradiation condition. 
much shorter lifetime. For this reason, the measuring method used for this study is almost insensitive to ${ }^{14} \mathrm{O}$.

\section{EXPERIMENTAL SET-UP}

The external beam transport line (BTL) of the Bern cyclotron was used for the measurements ${ }^{(3)}$. The cyclotron provides $18 \mathrm{-MeV}$ proton beams of variable intensity up to $150 \mu \mathrm{A}$. The BTL is equipped with an up-stream collimator, $X-Y$ steering magnets, two quadrupole doublets, a neutron shutter, two beam viewers with Faraday cups for measuring the current and a beam dump. The beam line ends in a separate bunker. A schematic view of the cyclotron and the BTL is presented in Figure 2. For the measurements reported in this study, the beam dump was replaced by a $400-\mu \mathrm{m}$ thick aluminium exit window.

The laboratory is equipped with high-sensitivity detectors for the continuous measurement of radioactivity in exhausted air $^{(4)}$. These detectors are installed inside the main exhaust pipe of the facility, a few metres before the chimney. They consist of two large surface proportional counters with an active area of $1000 \mathrm{~cm}^{2}$. The detectors are filled with argonmethane for low background counting rate. They are calibrated for ${ }^{18} \mathrm{~F}$, which is daily produced for the synthesis of PET radiotracers. Since the decays of ${ }^{11} \mathrm{C}$ and ${ }^{13} \mathrm{~N}$ were considered in this experiment, the scaling factor $f$ given by the manufacturer ${ }^{(4)}$ was applied. It was determined to be 0.6 for ${ }^{13} \mathrm{~N}$. Although the maximum positron decay energies for these two radionuclides are slightly different, a single scaling factor was considered. This represents a good approximation for this study and was taken into account in the estimation of the uncertainty. The air monitoring system provides diverse estimators of the exhausted air contamination. In this experiment, the so-called chimney short emission value (CSEV) was used. It is computed on the basis of the measured activity per volume (in $\mathrm{kBq} \mathrm{m}^{-3}$ ) and of the air flow (in $\mathrm{m}^{3} \mathrm{~h}^{-1}$ ). The CSEV is expressed in $\mathrm{kBq} \mathrm{h}^{-1}$ and is updated every minute. The ventilation system runs continuously and was set at 5.5 air changes per hour in the BTL bunker (volume $63 \mathrm{~m}^{3}$ ). For the whole facility, $\sim 20000 \mathrm{~m}^{3} \mathrm{~h}^{-1}$ of air was exhausted to the atmosphere. Due to the transit time of the air in the ventilation system, radioactivity produced in the air of the BTL bunker was measured with a delay of $\sim 2 \mathrm{~min}$.

For the full proton range measurement, the intensity of extracted current was measured with a movable stopper made of aluminium. The movement of the

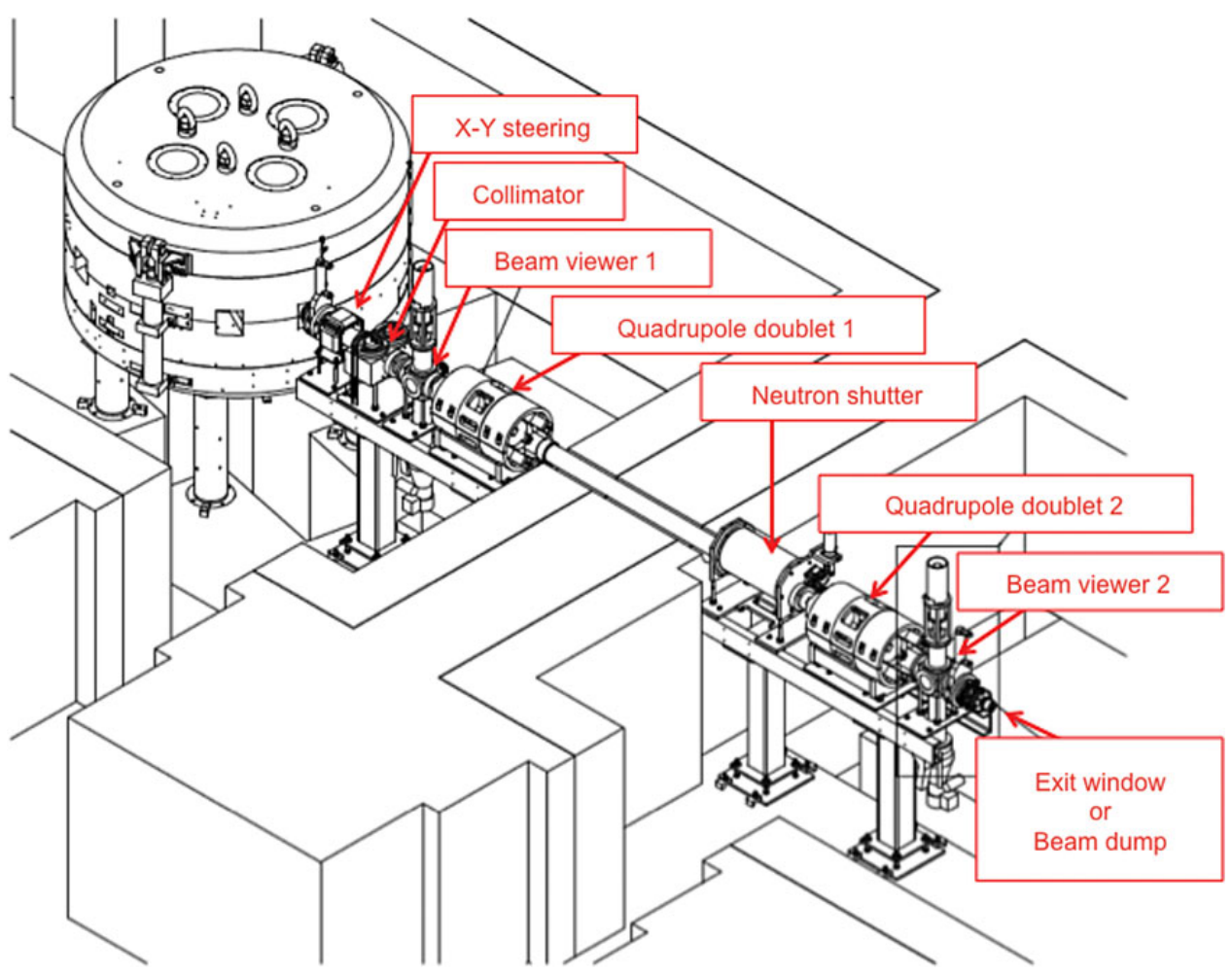

Figure 2. Schematic view of the Bern cyclotron and its beam transport line. 


\section{S. BRACCINI ET AL.}

stopper was automatised so that the beam could be extracted into air right after the measurement of the intensity. This system is shown in Figure 3. In this way, protons were extracted into air and fully stopped inside the bunker. The range of a $15-\mathrm{MeV}$ proton

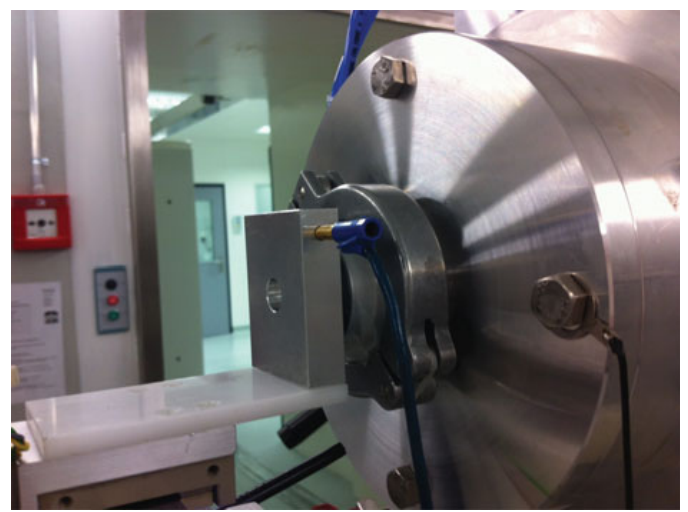

Figure 3. Motorised movable stopper for beam current measurements. beam in dry air calculated by means of SRIM was found to be $2.60 \mathrm{~m}$. The distance between the aluminium exit window and the bunker wall is $>3 \mathrm{~m}$ so that the beam is fully stopped in air. In the case of the measurement with the beam dump, protons travelled in air at a distance of $1.50 \mathrm{~m}$ and were stopped by a $20-\mathrm{mm}$ thick aluminium plate. This configuration is shown in Figure 4. The current measured on the beam dump corresponds to the beam intensity, since the fraction of protons undergoing nuclear reactions or large angle scattering effects is negligible. Each beam extraction in both irradiation conditions was followed by $\sim 45 \mathrm{~min}$ of data taking with the air monitoring system. The next irradiation was performed only when the activity reached again the level of the background, as presented in Figure 5.

\section{DATA ANALYSIS}

Data recorded by the high-sensitivity proportional counters installed in the main exhaust allowed estimating the radioactivity produced during the irradiation. As shown in Figure 1, the contribution due to ${ }^{14} \mathrm{O}$ is almost negligible and was considered only for

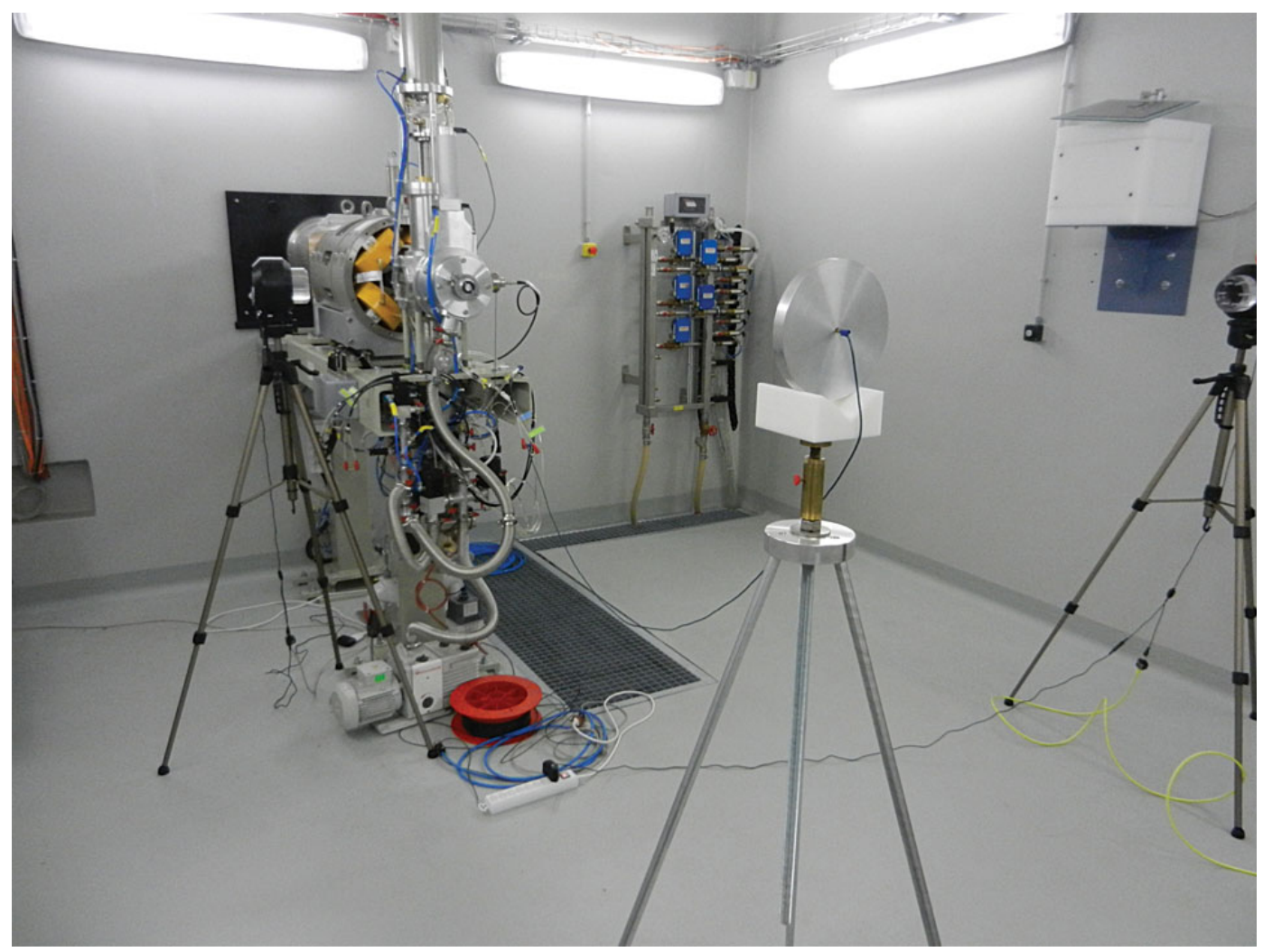

Figure 4. Set-up for the measurement with a beam dump at the distance of $1.5 \mathrm{~m}$. 


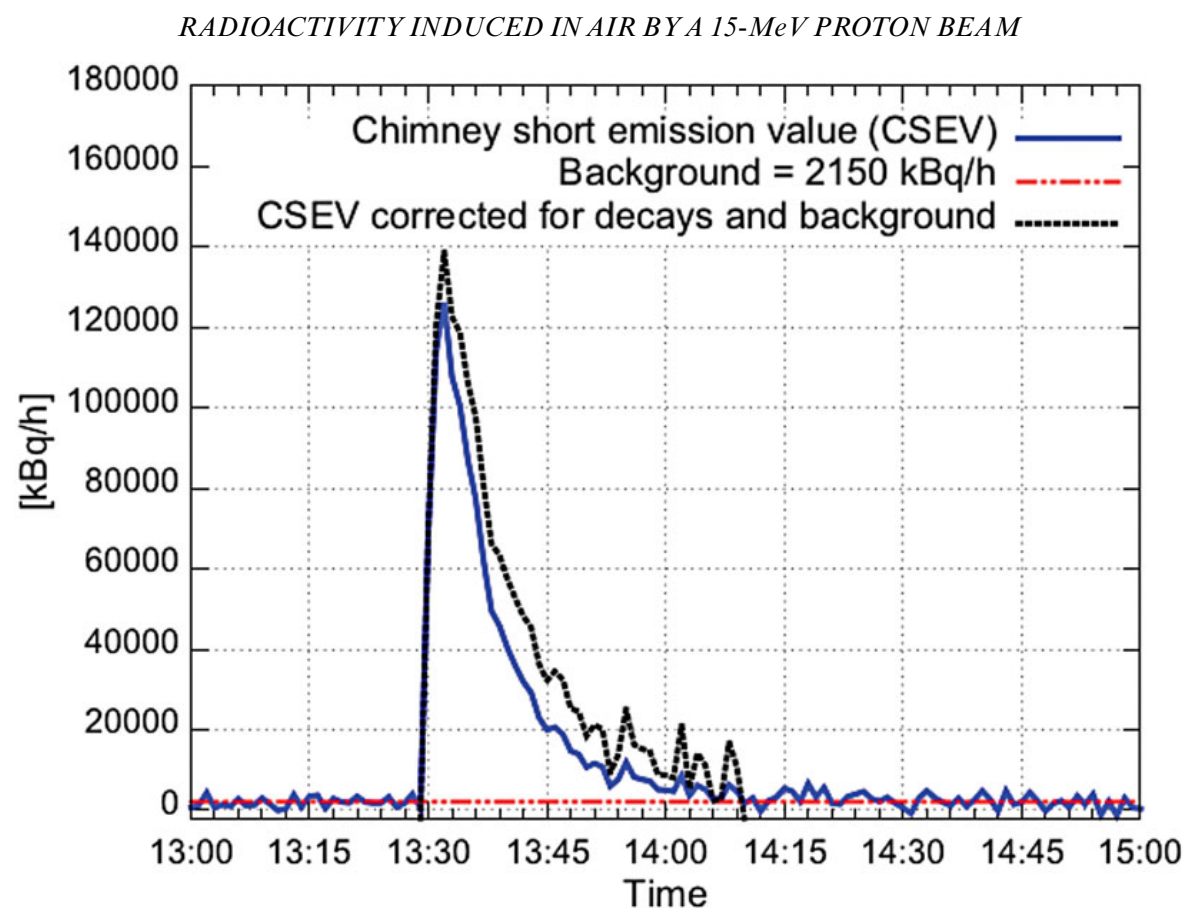

Figure 5. The CSEVs as a function of time for one irradiation (solid line) and the corresponding estimated background (dashed-dotted line). To calculate the produced activities at EOB $A_{\mathrm{i}}\left(t_{0}\right)$, the CSEVs were corrected for decay and background (dotted line). The EOB happened 2 min before the rise of the signal.

the estimation of the uncertainty. Since no other experimental activities were carried out in the whole facility during the measurements, no contamination due to other isotopes was possible. The activities $A_{\mathrm{C}}$ and $A_{\mathrm{N}}$ - corresponding to ${ }^{11} \mathrm{C}$ and ${ }^{13} \mathrm{~N}$, respectively - are therefore considered for the data analysis. Considering the decay constants $\lambda_{\mathrm{C}}$ and $\lambda_{\mathrm{N}}$, the time evolution of $A_{\mathrm{C}}$ and $A_{\mathrm{N}}$ is given by

$$
\begin{aligned}
& A_{\mathrm{C}}(t)=A_{\mathrm{C}}\left(t_{0}\right) \mathrm{e}^{-\lambda_{\mathrm{C}} t}, \\
& A_{\mathrm{N}}(t)=A_{\mathrm{N}}\left(t_{0}\right) \mathrm{e}^{-\lambda_{\mathrm{N}} t},
\end{aligned}
$$

where $t_{0}$ corresponds to the end of bombardment (EOB). The total induced activity at EOB $A_{\mathrm{tot}}\left(t_{0}\right)$ is given by

$$
A_{\text {tot }}\left(t_{0}\right)=A_{\mathrm{C}}\left(t_{0}\right)+A_{\mathrm{N}}\left(t_{0}\right),
$$

and was obtained through the measurements of the CSEVs performed at successive time instants $t_{\mathrm{i}}$, as presented in Figure 5 for one of the irradiations. Due to the continuous air flow in the ventilation system, signals different from background are recorded up to $\sim 40$ min after EOB.

Since the total activity was measured, the contributions of ${ }^{11} \mathrm{C}$ and ${ }^{13} \mathrm{~N}$ were estimated on the basis of the calculated values $A_{\mathrm{C}}^{\text {calc }}\left(t_{0}\right)$ and $A_{\mathrm{N}}^{\text {calc }}\left(t_{0}\right)$. The factor $k_{\mathrm{N}}$ is introduced and represents the fraction of the total activity due to ${ }^{13} \mathrm{~N}$ at $t_{0}$ :

$$
k_{\mathrm{N}}=\frac{A_{\mathrm{N}}^{\text {calc }}\left(t_{0}\right)}{A_{\mathrm{C}}^{\text {calc }}\left(t_{0}\right)+A_{\mathrm{N}}^{\text {calc }}\left(t_{0}\right)} .
$$

The factor $k_{\mathrm{N}}$ is equal to 0.24 and 0.31 for the full proton range and for protons stopped at $1.5 \mathrm{~m}$, respectively. Small variations of $k_{\mathrm{N}}$ due to the irradiation time were taken into account. This leads to the formula for the activity $A_{i}\left(t_{i}\right)$ corresponding to the $i$ th measurement of the radioactivity released in air:

$$
A_{i}\left(t_{i}\right)=A_{i}\left(t_{0}\right)\left(k_{\mathrm{N}} \mathrm{e}^{-\lambda_{\mathrm{N}} t_{i}}+\left(1-k_{\mathrm{N}}\right) \mathrm{e}^{-\lambda_{C} t_{i}}\right) .
$$

The total produced activity $A_{\text {tot }}\left(t_{0}\right)$ is the sum of all the activities $A_{i}\left(t_{0}\right)$ :

$$
\begin{aligned}
& A_{\mathrm{tot}}\left(t_{0}\right)=\sum_{i} A_{i}\left(t_{0}\right) \\
& =f \sum_{i} \frac{\epsilon_{i}}{k_{\mathrm{N}} \mathrm{e}^{-\lambda_{\mathrm{N}} t_{i}}+\left(1-k_{\mathrm{N}}\right) \mathrm{e}^{-\lambda_{C} t_{i}}},
\end{aligned}
$$

where $\varepsilon_{i}$ is the CSEV multiplied by the time interval 


\section{S. BRACCINI ET AL.}

Table 2. Summary of the measurements performed in the two irradiation conditions together with the corresponding calculated values.

\begin{tabular}{ccccc}
\hline$\#$ & $\begin{array}{c}\text { Beam } \\
\text { current } \\
{[\mathrm{nA}]}\end{array}$ & $\begin{array}{c}\text { Irradiation } \\
\text { time [s] }\end{array}$ & $\begin{array}{c}A_{\text {tot }}\left(t_{0}\right) \\
{[\mathrm{MBq}]}\end{array}$ & $A^{\text {calc }}[\mathrm{MBq}]$ \\
& & & \\
\hline
\end{tabular}

\begin{tabular}{ccrrrr}
\multicolumn{3}{c}{ Full proton range } & & & \\
1 & 120 & 30 & $12.28 \pm 0.88$ & $14.15 \pm 2.37$ \\
2 & 260 & 15 & $13.74 \pm 0.95$ & $15.35 \pm 2.57$ \\
3 & 280 & 20 & $22.66 \pm 1.50$ & $22.00 \pm 3.69$ \\
4 & 260 & 20 & $17.78 \pm 1.11$ & $20.43 \pm 3.42$ \\
\multicolumn{2}{c}{ Protons stopped at } & $1.5 \mathrm{~m}$ & & & \\
1 & 370 & 10 & $10.37 \pm 0.76$ & $10.44 \pm 1.43$ \\
2 & 170 & 20 & $10.35 \pm 0.77$ & $9.55 \pm 1.31$ \\
3 & 220 & 30 & $19.11 \pm 1.27$ & $18.48 \pm 2.53$ \\
4 & 260 & 30 & $22.07 \pm 1.48$ & $21.84 \pm 2.99$
\end{tabular}

$A^{\text {calc }}$ corresponds to the contributions of ${ }^{11} \mathrm{C}$ and ${ }^{13} \mathrm{~N}$.

Table 3. Experimental results compared with calculations. Total activities per beam current and irradiation time are reported for full proton range and protons stopped at the distance of $1.5 \mathrm{~m}$. $\mathrm{A}^{\text {calc }}$ corresponds to the contributions of ${ }^{11} \mathrm{C}$ and ${ }^{13} \mathrm{~N}$.

$A_{\text {tot }}\left(t_{0}\right) /(I \cdot t)\left[\mathrm{MBq}(\mathrm{nA} \mathrm{s})^{-1}\right] \quad A^{\text {calc }} /(I \cdot t)\left[\mathrm{MBq}(\mathrm{nA} \mathrm{s})^{-1}\right]$

Full proton range

$(3.60 \pm 0.48) \times 10^{-3}$

Protons stopped at $1.5 \mathrm{~m}$

$(2.89 \pm 0.37) \times 10^{-3}$

$(3.93 \pm 0.66) \times 10^{-3}$

$(2.81 \pm 0.39) \times 10^{-3}$

of one minute and $f=0.6$ is the scaling factor of the proportional counters for ${ }^{11} \mathrm{C}$ and ${ }^{13} \mathrm{~N}$.

For the two different irradiation conditions, four measurements were performed. They are reported in Table 2 together with the calculated values $A^{\text {calc }}$, which include the contributions of ${ }^{11} \mathrm{C}$ and ${ }^{13} \mathrm{~N}$. Good agreement is found between measurements and calculations.

According to Equation (3), the produced activity $A(t)$ can be linearly approximated for $t \ll 1 / \lambda$, where $t$ denotes the irradiation time. Since this condition was fulfilled in the reported measurements, the average values of $A_{\text {tot }}\left(t_{0}\right)$ per beam current $I$ and irradiation time $t$ were calculated for both irradiation conditions.

The results compared with the corresponding values of $A^{\text {calc }} /(I \cdot t)$ are presented in Table 3 .

The main source of uncertainty is the precision of the air monitoring system, which is estimated by the manufacturer to be $20 \%$ of the measured value. Statistical uncertainties are significant and account for up to $\sim 30 \%$ of the total uncertainties. Other relevant sources of uncertainty are the beam current $(\simeq 10 \%)$, the scaling factor of the proportional counters $(\simeq 10 \%)$, the irradiation time $(\simeq 5 \%)$, the $k_{\mathrm{N}}$ factor $(\simeq 5 \%)$ and the presence of a residual contribution due to ${ }^{14} \mathrm{O}(\simeq 4 \%)$.

At the cyclotron laboratory in Bern, the alarm threshold for air contamination is set at $70 \mathrm{kBq} \mathrm{m}^{-3}$ at the main exhaust and, according to the Swiss regulations, a maximum of $784 \mathrm{MBq}$ can be exhausted per week. If the alarm threshold is overcome, the ventilation of the whole facility shuts down, provoking severe consequences for the good manufacturing practice (GMP) radiopharmaceutical production and for radiation protection procedures. For these reasons, extreme care is needed when research activities requiring the use of the proton beam extracted into air are performed. As an example, the extraction of $500 \mathrm{nA}$ for 60 s producing $\sim 100 \mathrm{MBq}$ of activity is sufficient to trigger the alarm.

\section{CONCLUSIONS}

The activity induced by a $15-\mathrm{MeV}$ proton beam extracted into air was measured and calculated for two irradiation conditions. In the former, protons were allowed to travel for their full range in air. In the latter, the beam was stopped at the distance of $1.5 \mathrm{~m}$ by an aluminium beam dump.

Calculations were based on excitation functions available in the literature. The experimental work was performed with the beam transport line of the 18$\mathrm{MeV}$ cyclotron at the Bern University Hospital (Inselspital). The produced radioactivity was measured by means of large surface proportional counters located at the main exhaust of the cyclotron laboratory. Proton beams in the current range of $120-370$ $\mathrm{nA}$ were extracted into air for a duration of $10-30 \mathrm{~s}$.

In both irradiation conditions, the main produced isotopes were ${ }^{11} \mathrm{C},{ }^{13} \mathrm{~N}$ and ${ }^{14} \mathrm{O}$. Due to the transit time of the air flow between the beam line bunker and the main exhaust of $\sim 2 \mathrm{~min}$, the contribution to the measurements given by the short-lived ${ }^{14} \mathrm{O}$ was negligible. The experimental results are found to be in good agreement with the calculations for ${ }^{11} \mathrm{C}$ and ${ }^{13} \mathrm{~N}$ within the total uncertainties. For irradiation times much shorter than ${ }^{11} \mathrm{C}$ and ${ }^{13} \mathrm{~N}$ lifetimes, the induced total activities per beam current and irradiation time were measured to be $(3.60 \pm 0.48) \times 10^{-3} \mathrm{MBq}$ $(\mathrm{nA} \mathrm{s})^{-1}$ and $(2.89 \pm 0.37) \times 10^{-3} \mathrm{MBq}(\mathrm{nA} \mathrm{s})^{-1}$, for the former and for the latter irradiation conditions, respectively.

The extraction of proton beams into air requires particular care, especially if performed in PET radiopharmaceutical production centres. The produced activity may induce alarms, ventilation shut downs and radiation protection issues with direct consequences for the production. The results reported in this study can be used for the assessment of radioactivity induced in air by proton beams in the energy range of PET cyclotrons. 


\section{ACKNOWLEDGEMENTS}

The authors would like to acknowledge contributions from LHEP engineering and technical staff. The authors sincerely thank Dr Jonas Knüsel and Dr Cyril Topfel from SWAN Isotopen AG for the support during the measurements and Dr I. Kreslo from LHEP for his constructive comments in reviewing this manuscript. The authors would like to gratefully acknowledge Vladislav Rozhkhov from the Kharkov Institute of Physics and Technology for his help in the construction and test of some parts of the equipment.

\section{FUNDING}

This work was supported by the Albert Einstein Center for Fundamental Physics (AEC) of the University of Bern and by the Swiss National Science Foundation (SNSF).

\section{REFERENCES}

1. Braccini, S. et al. SWAN: a combined centre for radioisotope production, proton therapy and research in Bern. In:
Presented at the Workshop on Physics for Health in Europe, CERN, Geneva, Switzerland (2010).

2. Braccini, S. et al. The new Bern cyclotron laboratory for radioisotope production and research. In: Proceedings of the Second International Particle Accelerator Conference (IPAC2011), San Sebastian, Spain, 3618 (2011).

3. Braccini, S. The new Bern PET cyclotron, its research beam line, and the development of an innovative beam monitor detector. In: Proceedings of the 22nd International Conference on the Application of Accelerators in Research and Industry (CAARI 2012), Forth Worth, Texas, USA (2012).

4. Berthold Technologies $\mathrm{GmbH}$. Radiation protection for PET isotope centers (2008).

5. (2003) CRC Handbook of Chemistry and Physics, eightyfourth edn, CRC Press.

6. International Atomic Energy Agency. Experimental nuclear reaction data (EXFOR). IAEA. http://wwwpub.iaea.org. (accessed 19 June, 2014).

7. Ziegler, J. F. and Manoyan, J. M. The stopping of ions in compounds. Nucl. Instrum. Methods B 35, 215, SRIM2013.00 (1988). http://www.srim.org. (accessed 19 June, 2014).

8. International Atomic Energy Agency. Technical Reports Series No. 468: Cyclotron produced radionuclides: physical characteristics and production methods (2009).

9. Kovács, Z., et al. Cross section measurements using gas and solid targets for production of the positron-emitting radionuclide $O-14$. Radiochim. Acta 91, 185 (2003). 